Journal of Animal and Veterinary Advances 11 (18): 3469-3473, 2012

ISSN: $1680-5593$

(C) Medwell Journals, 2012

\title{
Characterization of MicroRNAs in Paragonimus westermani by Solexa Deep Sequencing and Bioinformatics Analysis
}

\author{
L. Ai, M.X. Chen, S.H. Chen, Y.N. Zhang, H. Li, Y.C. Cai, Y. Lu, \\ L.G. Tian, X.N. Zhou and J.X. Chen \\ National Institute of Parasitic Diseases, Chinese Center for Disease Control and Prevention, \\ Laboratory of Parasite and Vector Biology, Ministry of Health, \\ WHO Collaborating Center for Malaria, Schistosomiasis and Filariasis, \\ 200025 Shanghai, P.R. China
}

\begin{abstract}
MicroRNAs (miRNAs) are small non-coding RNA which generate from large hairpin precursors and play important role on post-transcriptional regulators of target genes. Although, many individual miRNAs have recently been extensively studied, there has been very little research on miRNA transcriptomes in trematode. By the method of using high throughput Solexa sequencing technology, 11.02 million clean reads have been obtained of Paragonimus westermani. Among the clean reads, 1.14 million ones (10.37\%) were perfectly mapped onto the $S$.japonicum genome which included $20,322(0.69 \%)$ unique sequences. A number of $9,492,613$ $(86.17 \%)$ reads had no match with public databases and marked as un-annotated RNAs. Nucleotide bias analysis found that the known miRNAs showed high bias and the guanine was the dominant nucleotide, particularly at the 2nd and 23rd positions which were almost at the beginning and end of conserved miRNAs. Three novel miRNA corresponding to 18 precursors were predicted. To the knowledge, this is the first report of miRNA profiles in $P$. westermani which will contribute to better understanding of the complex biology of this zoonotic parasite. The reported data of $P$. westermani miRNAs should provide valuable references for miRNA studies of closed related zoonotic trematode.
\end{abstract}

Key words: Paragonimus westermani, microRNAs (miRNAs), Solexa deep sequencing, novel, China

\section{INTRODUCTION}

Paragonimus westermani which parasitize in the lung of human with various kinds of mammal animals as definitive hosts. This trematode causes paragonimiasis in many Asian countiries such as Japan, Korea, China, etc. where culturally people eat raw or undercooked freshwater crab or crayfishes infected with infective metacercariae (Mukae et al., 2001; Liu et al., 2008; Kim et al., 2009). Currently, paragonimiasis has been recognized as a serious food-borne disease with the symptom as lung cancer or tuberculosis which always led to misdiagnosis (Lane et al., 2009; Sohn et al., 2009; Ikehara et al., 2010).

Although, the research of classification, pathogenicity, immunologic mechanism, etc. on $P$. westermani have been carried out for many years (Zhao et al., 2007; Liu et al., 2008; Devi et al., 2010), increasing knowledge of microRNAs (miRNAs) is able to reveal riboregulators throughout different animal phyla
(Grimson et al., 2008), uncover developmental genetic switches (Flynt and Lai, 2008) and explore novel biomarkers for disease (Zhang, 2008; Mo, 2012).

The microRNAs (miRNA) have been found in many organisms such as viruses, plants and mammals (Grant-Downton et al., 2009; Dolken et al., 2010; Su et al., 2011 ) which are non-coding small RNA molecules with the length of 18-24 nucleotides and play essential roles in gene expressions of living organisms (Mead and Tu, 2008; Reddy et al., 2009). In the miRNA research of parasites, miRNAs have been identified in model organism Caenorhabditis elegans as well as some zoonotic parasites such as Schistosoma japonicum, Clonorchis sinensis, Toxoplasma gondii, Trichinella spiralis and Angiostrongylus cantonensis (Wang et al., 2010; Xu et al., 2010; Warf et al., 2011; Chen et al., 2011a, b). However, there have been no reports of miRNAs in $P$. westermani.

Therefore, the aim of the present study was to investigate the expression profile of miRNAs in adult

Corresponding Author: J.X. Chen, National Institute of Parasitic Diseases, Chinese Center for Disease Control and Prevention, 200025 Shanghai, The People's Republic of China 
P. westermani and to predict potential novel miRNAs by Solexa deep sequencing method and bioinformatics analysis.

\section{MATERIALS AND METHODS}

Total RNA preparation and sequencing: The adult $P$. westermani were collected from lung of the artificial infected dog in the lab and the infected metacercaria from freshwater crab in Zhejiang province which were used to feed the dog. After harvesting, the parasites were transferred immediately to sterile physiological saline $\left(37^{\circ} \mathrm{C}\right)$ in a sterile beaker and washed 5 times to remove contaminants from the host. One adult was cut into pieces and transferred to RNase-free $1.5 \mathrm{~mL}$ screw-top cryotube containing RNAlater (Sigma). Then, the sample was kept at $4^{\circ} \mathrm{C}$ for overnight and stored at $-70^{\circ} \mathrm{C}$ until use. The taxonomic identity of the trematode was confirmed as P. westermani by Polymerase Chain Reaction (PCR) amplification and subsequent sequence analysis of the mitochondrial cytochrome $\mathrm{c}$ oxidase subunit 1 gene ( $\operatorname{cox} 1)$ (Park et al., 2003).

Trizol (Invitrogen) was used to prepare total RNA in terms of the protocol provided by the manufacturer. RNA concentration and purity were evaluated photometrically by measuring the absorbance at 260 and $280 \mathrm{~nm}$, through NanoDrop ND-1000 spectrophotometer (Nanodrop Technologies, Wilmington, DE) and Agilent 2100 Bioanalyzer (Agilent Technologies, Palo Alto, CA). After obtaining pure RNA, it stored at $-70^{\circ} \mathrm{C}$ (Lau et al., 2001; Xu et al., 2010). About $20 \mu \mathrm{g}$ total RNA was separated by electrophoresis on a Novex $15 \%$ urea gel (Invitrogen Co., Ltd.) with RNA in the region of 18-30 nt purified. Next, a Reverse Transcription PCR (RT-PCR) kit (Invitrogen) was used for reverse transcription of the small RNAs to form complementary DNA (cDNA). Finally, the cDNA was then sequenced with a Solexa sequencer at Huada Genomics Institute Co., Ltd. China.

High-throughput sequencing and computational analysis: Data analysis refered as earlier ( $\mathrm{Xu}$ et al., 2010; Chen et al., $2011 \mathrm{a}, \mathrm{b}$ ). Firstly, adaptors, reads smaller than $18 \mathrm{nt}$ and low quality sequences were trimmed from the raw data. Next, the reads were searched in GenBank Rfam Database (Version 10.1) to remove non-coding RNAs including rRNA, tRNA, snRNA, snoRNA and other ncRNAs. RepeatMasker (http://www.repeatmasker.org) was used to eliminate repetitive sequences in the clean reads. Reads of $18-30 \mathrm{nt}$ were mapped onto the genome of S. japonicum (http://lifecenter.sgst.cn/schistosoma/cn) using Short Oligo nucleotide Analysis Package Software (SOAP, http://soap.genomics.org.cn) (Bartel, 2004;
Rhoades et al., 2002). Further bioinformatics analysis including unique reads screening, candidate precursors, inverted repeats, family distribution of conserved miRNA and the nucleotide bias were performed as described by Xue et al. (2008).

\section{RESULTS AND DISCUSSION}

High throughput sequencing technologies provide more convenient assay to detect known and novel miRNAs which can also open doors to directly show differences in expression levels. Given that miRNAs play essential roles in gene expression, research of miRNAs in parasites can prob mechanisms that the parasites respond to environmental and developmental signals through miRNA-mediated gene expression (Liu et al., 2010). Therefore, the present study examined the expression profiles of miRNAs in $P$. westermani by Solexa deep sequencing combined with bioinformatics analysis.

There were 12.99 million reads yielded with Solexa sequencing. After filtering low quality tags including $5^{\prime}$ and $3^{\prime}$ adaptors and adaptor-adaptor ligation, a total of 12.87 million reads with high quality were obtained. Length distribution analysis showed that most reads distributed among 20-23 nt. The highest percentage was $16.03 \%$ of $22 \mathrm{nt}$ reads followed by $15.08 \%$ of $23 \mathrm{nt}$ reads (Fig. 1). After removing reads smaller than $18 \mathrm{nt}(12.72 \%)$, a total of 11.02 million clean reads being obtained. Among the clean reads, 1.14 million ones $(10.37 \%)$ were perfectly mapped onto the $S$. japonicum genome which included $20,322(0.69 \%)$ unique sequences. However, it was found that reads matched to the reference genome of $S$. japonicum was very low. However, it can reach as high as $70.5 \%$ or higher in some other species (Xue et al., 2008). The most possible reason for the phenomenon might be that the reference genome was $S$.japonicum

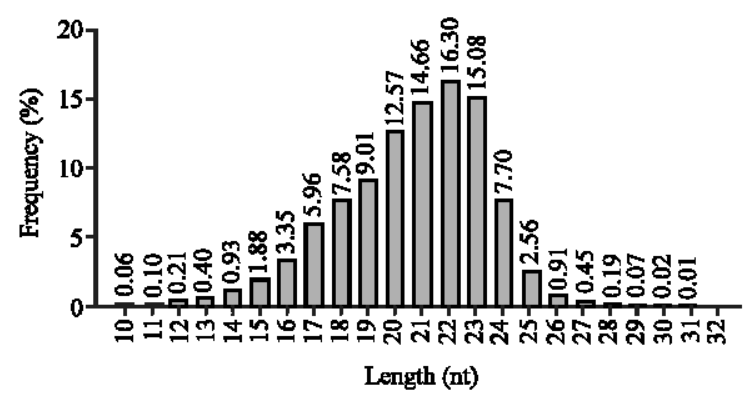

Fig. 1: Length distribution analysis of small RNAs of Paragonimus westermani. Analysis of the 12.87 million reads with high qualities after filtering low quality tags, $5^{\prime}$ and $3^{\prime}$ adaptor and contamination formed by adaptor-adaptor ligation 


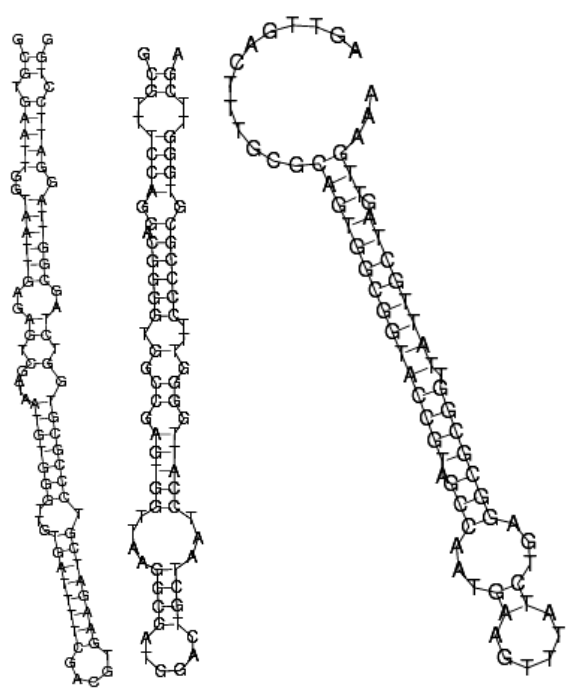

Fig. 2: Predicted structures of the novel miRNA precursors of Paragonimus westermani evaluated by RNA-Fold Software

Table 1: Detailed classification of reads of Paragonimus westermani

\begin{tabular}{lrr}
\hline & $P$ westermani & \\
& Total sRNAs (\%) & Unique sRNAs (\%) \\
\hline Total & $11015886(100.00)$ & $2949024(100.00)$ \\
miRNA & $568896(5.16)$ & $219(0.01)$ \\
rRNA & $761689(6.91)$ & $36143(1.23)$ \\
snRNA & $1532(0.01)$ & $690(0.02)$ \\
SnoRNA & $2170(0.02)$ & $314(0.01)$ \\
tRNA & $165276(1.50)$ & $7368(0.25)$ \\
Unann & $9492613(86.17)$ & $2902039(98.41)$ \\
\hline
\end{tabular}

genome rather than $P$. westermani because the $P$. westermani genome is not available currently. For the same reason, there were only three novel miRNAs predicted in adult $P$. westermani (Fig. 2). When the genome of $P$. westermani or other closely related trematodes become available, more miRNAs will be found in $P$. westermani.

Among the clean reads, 930,667 (8.44\%) were found as rRNA, tRNA, snRNA and snoRNA when searched against Rfam Database (Table 1). Two types of repeats of LINE/RTE:0 andLINE/RTE: 1 with 1668 reads (474 unique ones). The percentage of known miRNA was 568,898 total reads corresponding to 221 unique ones focusing on 18 conserved miRNAs. In addition to the known miRNAs, rRNA and repeats mentioned above, a number of $9,492,613(86.17 \%)$ reads had no match with public databases and marked as un-annotated RNAs. Among them, 2,902,039 were unique reads (Fig. 3).

Some kinds of miRNAs were expressed with high predominance. The miR-71 had the most abundant reads with a percentage of $68.80 \%$ (391,382 reads). The miR-71 occupied most portions of the total reads which indicated

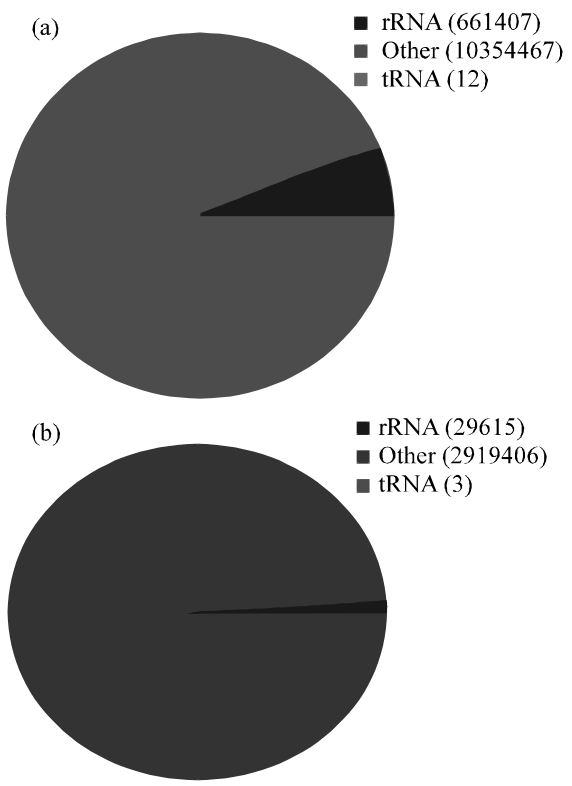

Fig. 3: Coverage of small RNAs in Paragonimus westermani by Solexa deep sequencing. a) Total reads; b) Unique reads

Table 2: Expressed miRNAs in Paragonimus westermani

\begin{tabular}{lr}
\hline miR name & Express \\
\hline sja-miR-71 & 391382 \\
sja-bantam & 67557 \\
sja-miR-71b-5p & 38382 \\
sja-miR-2a-3p & 24809 \\
sja-miR-7-5p & 18178 \\
sja-let-7 & 12321 \\
sja-miR-277 & 7284 \\
sja-miR-61 & 3667 \\
sja-miR-10-5p & 2719 \\
sja-miR-219-5p & 1325 \\
sja-miR-2b-3p & 625 \\
sja-miR-124-3p & 261 \\
sja-miR-2e-3p & 242 \\
sja-miR-2c-3p & 83 \\
sja-miR-2d-3p & 50 \\
sja-miR-36-3p & 6 \\
sja-miR-307 & 2 \\
sja-miR-3488 & 2 \\
\hline
\end{tabular}

that the miR-71 was essential for the survival of the trematode. This phenomenon has also been shown in S. japonicum, Fasciola sp. as well as Taenia cestode (Xue et al., 2008; Ai et al., 2012; Xu et al., 2012). In addition to the miR-71, some other miRNAs had particularly high copies in $P$. westermani such as bantam, miR-2a-3p, miR-7-5p. bantam with a percentage of $11.88 \%$ with 67,557 reads which was also with high copy in S.japonicum (Xue et al., 2008). However, let-7 family was the biggest miRNA family among the conserved miRNAs in C. elegans and C. sinensis (Reinhart et al., 2000; $\mathrm{Xu}$ et al., 2010). The let- 7 or its family members were only $2.17 \%$ with 12,321 reads identified in $P$. westermani in the present study (Table 2). 
Table 3: Positions and rate of sRNA in Paragonimus westermani

\begin{tabular}{|c|c|c|c|c|c|c|}
\hline $\begin{array}{l}\text { Position o } \\
\text { SRNA }\end{array}$ & $\mathrm{A}(\%)$ & $\mathrm{U}(\%)$ & $\mathrm{C}(\%)$ & $\mathrm{G}(\%)$ & $\mathrm{A}+\mathrm{U}(\%)$ & $5(20)$ \\
\hline 1 & 1.190 & 95.750 & 0.002 & 3.047 & 96.950 & 0.000 \\
\hline & 2.170 & 0.000 & 4.320 & 95.520 & 2.170 & 3.050 \\
\hline & 94.460 & 0.015 & 1.200 & 4.320 & 97.480 & 5.520 \\
\hline & 89.880 & 0.350 & 6.670 & 3.090 & 90.230 & 9.770 \\
\hline & 93.310 & 2.390 & 1.200 & 3.100 & 95.700 & 4.300 \\
\hline 6 & 1.140 & 4.250 & 0.070 & 94.540 & 5.390 & 94.610 \\
\hline & 92.100 & 0.350 & 5.220 & 2.340 & 92.450 & 7.550 \\
\hline 8 & 2.040 & 1.200 & 93.700 & 3.060 & 240 & 96.760 \\
\hline & 6.660 & 20.5 & & 72.430 & 27. & 72.780 \\
\hline 10 & 73.870 & 16.22 & 0.066 & 9.840 & 90.090 & 9.906 \\
\hline 11 & 1.200 & 77.940 & 3.060 & 17.800 & 79.140 & 20.860 \\
\hline 12 & 16.590 & 6.720 & 1.200 & 75.490 & 23.310 & 76.690 \\
\hline 13 & 0.002 & 7.420 & 2.440 & 90.140 & 22 & 92.580 \\
\hline 14 & 4.320 & 92.9 & 0 & 0.4 & & 2.750 \\
\hline 15 & 85.850 & 4.340 & 0.350 & 9.456 & 90.190 & 9.810 \\
\hline 16 & 10.240 & 3.060 & 1.140 & 85.560 & 13.300 & 86.700 \\
\hline 17 & 0.017 & 94.830 & 0.900 & 4.253 & 94.850 & 5.150 \\
\hline 18 & 0.000 & & & 93.3 & & 95.410 \\
\hline 19 & 90.12 & 5. & 0. & 3.0 & 96.0 & 3.960 \\
\hline 20 & 43 & 5.5 & 1.540 & 88.580 & 9.880 & 90.120 \\
\hline 21 & 88.530 & 5.680 & 0.000 & 5.740 & 94.260 & 5.740 \\
\hline 22 & 0.990 & 84.460 & 13.260 & 1.290 & 85.450 & 14.550 \\
\hline 23 & 1.030 & & & 98.940 & 1.050 & 98.940 \\
\hline Ave & 33.060 & 23.240 & 6.170 & 37.620 & 56.420 & 39.460 \\
\hline
\end{tabular}

Analysis of the 221 unique reads of known miRNAs showed that $\mathrm{G}$ was the dominant nucleotide in mature miRNAs with the average percentage of $37.62 \%$ and showed high percentage at the 23rd nucleotide of $98.94 \%$. The highest percentage of $(\mathrm{G}+\mathrm{C})$ was found at the $23 \mathrm{rd}$ positions with a percentage of $98.94 \%$. The U was most often used as the first nucleotide in miRNAs of $P$. westermani with an average percentage of $95.75 \%$. $(\mathrm{A}+\mathrm{U})$ was found in most reads with a percentage of $56.42 \%$ in average (Table 3 ).

\section{CONCLUSION}

The present study represents the miRNAs characterization of $P$. westermani which will be useful to help understanding the complex biology of this trematode and to explore effective control methods for paragonimosis.

\section{ACKNOWLEDGEMENTS}

This research is supported in part by the Shanghai Postdoctoral Sustentation Fund (Grant No. 12R21416500), China Postdoctoral Science Foundation (Grant No. 2012M520353), National S\&T Major Program (Grant No. 2008ZX10004-011,2009ZX10004-302,2009ZX10004-201), and National Key Technology R and D Program (Grant No. 2008BAI56B03, the Open Funds of the State Key Laboratory of Veterinary Etiological Biology, Lanzhou Veterinary Research Institute, Chinese Academy of Agricultural Sciences (Grant Nos. SKLVEB2009KFKT008,
SKLVEB2009KFKT014,SKLVEB2011KFKT004,SKLVEB2010KFKT009, SKLVEB2010KFKT010 and SKLVEB2011KFKT010).

\section{REFERENCES}

Ai, L., M.J. Xu, M.X. Chen, Y.N. Zhang and S.H. Chen et al., 2012. Characterization of microRNAs in Taenia saginata of zoonotic significance by Solexa deep sequencing and bioinformatics analysis. Parasitol. Res., 110: 2373-2378.

Bartel, D.P., 2004. MicroRNAs: Genomics, biogenesis, mechanism and function. Cell, 116: 281-297.

Chen, M.X., L. Ai, M.J. Xu, R.L. Zhang and S.H. Chen et al., 2011a. Angiostrongylus cantonensis: Identification and characterization of microRNAs in male and female adults. Exp. Parasitol., 128: 116-120.

Chen, M.X., L. Ai, M.J. Xu, S.H. Chen and Y.N. Zhang et al., 2011b. Identification and characterization of microRNAs in Trichinella spiralis by comparison with Brugia malayi and Caenorhabditis elegans. Parasitol. Res., 109: 553-558.

Devi, K.R., K. Narain, T. Agatsuma, D. Blair and M. Nagataki et al., 2010. Morphological and molecular characterization of Paragonimus westermani in northeastern India. Acta Tropica, 116: 31-38.

Dolken, L., A. Krmpotic, S. Kothe, L. Tuddenham and M. Tanguy et al., 2010. Cytomegalovirus microRNAs facilitate persistent virus infection in salivary glands. PLoS Pathog, 10.1371/journal.ppat.1001150.

Flynt, A.S. and E.C. Lai, 2008. Biological principles of microRNA-mediated regulation: Shared themes amid diversity. Nat. Rev. Genet., 9: 831-842.

Grant-Downton, R., G. Le Trionnaire, R. Schmid, J. Rodriguez-Enriquez and S. Hafidh et al., 2009. MicroRNA and tasiRNA diversity in mature pollen of Arabidopsis thaliana. BMC Genomics, 10.1186/14712164-10-643.

Grimson, A., M. Srivastava, B. Fahey, B.J. Woodcroft and H.R. Chiang et al., 2008. Origins and evolution of microRNAs and Piwi-interacting RNAs in animals. Nature, 455: 1193-1197.

Ikehara, M., Y. Komase, A. Morita, H. Yamaguchi and T. Yamamoto, 2010. Paragonimiasis in a person whose symptoms were shown 22 years after emigrating to Japan from Laos. J. Infect. Chemother., 16: $49-52$.

Kim, E.M., J.L. Kim, S.I. Choi, S.H. Lee and S.T. Hong, 2009. Infection status of freshwater crabs and crayfish with metacercariae of Paragonimus westermani in Korea. Korean J. Parasitol., 47: 425-426. 
Lane, M.A., M.C. Barsanti, C.A. Santos, M. Yeung, S.J. Lubner and G.J. Weil, 2009. Human paragonimiasis in North America following ingestion of raw crayfish. Clin. Infect. Dis., 49: e55-e61.

Lau, N.C., L.P. Lim, E.G. Weinstein and D.P. Bartel, 2001. An abundant class of tiny RNAs with probable regulatory roles in Caenorhabditis elegans. Science, 294: 858-862.

Liu, Q., F. Wei, W. Liu, S. Yang and X. Zhang, 2008. Paragonimiasis: An important food-borne zoonosis in China. Trends Parasitol., 24: 318-323.

Liu, Q., W. Tuo, H. Gao and X.Q. Zhu, 2010. MicroRNAs of parasites: Current status and future perspectives. Parasitol. Res., 107: 501-507.

Mead, E.A. and Z. Tu, 2008. Cloning, characterization and expression of microRNAs from the Asian malaria mosquito, Anopheles stephensi. BMC Genomics, 10.1186/1471-2164-9-244.

Mo, Y.Y., 2012. MicroRNA regulatory networks and human disease. Cell. Mol. Life Sci., 69: 3529-3531.

Park, G.M., K.I. Im and T.S. Yong, 2003. Phylogenetic relationship of ribosomal ITS2 and mitochondrial COI among diploid and triploid Paragonimus westermani isolates. Korean J. Parasitol., 41: 47-55.

Reddy, A.M., Y. Zheng, G. Jagadeeswaran, S.L. Macmil and W.B. Graham et al., 2009. Cloning, characterization and expression analysis of porcine microRNAs. BMC Genomics, 10.1186/1471-2164-1065.

Reinhart, B.J., F.J. Slack, M. Basson, A.E. Pasquinelli and G. Ruvkun et al., 2000. The 21-nucleotide let-7 RNA regulates developmental timing in Caenorhabditis elegans. Nature, 403: 901-916.

Rhoades, M.W., B.J. Reinhart, C.B. Burge, B. Bartel and D.P. Bartel, 2002. Prediction of plant microRNA targets. Cell., 110: 513-520.
Sohn, B.S., Y.J. Bae, Y.S. Cho, H.B. Moon and T.B. Kim, 2009. Three cases of paragonimiasis in a family. Korean J. Parasitol., 47: 281-285.

$\mathrm{Su}, \mathrm{Z}$., J. Xia and Z. Zhao, 2011. Functional complementation between transcriptional methylation regulation and post-transcriptional microRNA regulation in the human genome. BMC Genomics, 10.1186/1471-2164-12-S5-S15.

Wang, Z., X. Xue, J. Sun, R. Luo and X. Xu et al., 2010. An in-depth description of the small non-coding RNA population of Schistosoma japonicum schistosomulum. PLoS Negl. Trop. Dis.

Warf, M.B., W.E. Johnson and B.L. Bass, 2011. Improved annotation of $\mathrm{C}$. elegans microRNAs by deep sequencing reveals structures associated with processing by Drosha and Dicer. RNA, 17: 563-577.

$\mathrm{Xu}$, M.J., L. Ai, J.H. Fu, J.N. Alasdair and Q.Y. Liu et al., 2012. Comparative characterization of MicroRNAs from the liver flukes Fasciola gigantica and $F$. hepatica. PloS.

Xu, M.J., Q. Liu, A.J. Nisbet, X.Q. Cai and C. Yan et al., 2010. Identification and characterization of microRNAs in Clonorchis sinensis of human health significance. BMC Genomics, 10.1186/14712164-11-521.

Xue, X., J. Sun, Q. Zhang, Z. Wang, Y. Huang and W. Pan, 2008. Identification and characterization of novel microRNAs from Schistosoma japonicum. PloS, 10.1371/journal.pone.0004034.

Zhang, C., 2008. MicroRNomics: A newly emerging approach for disease biology. Physiol. Genomics, 33: $139-147$.

Zhao, Q.P., S.U. Moon, B.K. Na, S.H. Kim and S.H. Cho et al., 2007. Paragonimus westermani: Biochemical and immunological characterizations of paramyosin. Exp. Parasitol., 115: 9-18. 